\title{
Lung Diseases due to Nontuberculous Mycobacteria: Report of 5 Cases
}

\author{
Müjgan Güler, Abdullah Şimşek, Ruhsar Ofluoğlu, Ebru Ünsal, Nermin Çapan
}

Clinic of Chest Surgery, Atatürk Chest Diseases and Chest Surgery Training and Research Hospital, Ankara

\begin{abstract}
Nontuberculous mycobacteria (NTM) are commonly isolated from environmental sources such as water and soil. Lung disease due to NTM commonly occurs in structural lung disease. Here, we report five cases of NTM. We identified two cases of Mycobacterium abscessus, one of $M$. chelonae, one of $M$. gordoneae, and one of $M$. szulgai by polymerase chain reaction (PCR). One of the patients with M. abscessus, despite aggressive treatment, died, whereas the other was alive and had no clinical and radiological progression without treatment. Without treatment, the progression of disease was not seen in the case of $M$. chelonae and that of $M$. gordoneae. Clinicoradiological regression occurred in the case of $M$. szulgai that was treated with HRZE. For correct diagnosis and the successful treatment of NTM pulmonary disease, knowledge of clinical, radiological, and microbiological findings is important.
\end{abstract}

Keywords: Diagnosis and treatment, nontuberculous mycobacteria

Received Date: 07.01 .2013

Accepted Date: 24.08 .2013

Available Online Date: 13.02.2015

Address for correspondence:

Abdullah Şimşek, Clinic of Chest Surgery, Atatürk Chest Diseases and Chest Surgery Training and

Research Hospital, Ankara, Turkey

E-mail: abdullahsimsekl@yahoo.com.tr

is work is licensed under a Creative Commons Attribution-NonCommerci 4.0 International License.

DOI: 10.5152/ejp.2014.26086

-Available online at www.eurasianjpulmonol.com

\section{INTRODUCTION}

Unlike Mycobacterium tuberculosis, which is an obligate human pathogen with no environmental reservoir, nontuberculous mycobacteria (NTM) are commonly isolated from environmental sources such as water and soil, and until the second half of the previous century, occasional isolates of NTM were thus largely considered to be contaminants or colonizers (1-4). Lung disease due to NTM commonly occurs in structural lung disease such as chronic obstructive lung disease (COPD), bronchiectasis, cystic fibrosis, pneumoconiosis, prior tuberculosis (TB), pulmonary alveolar proteinosis, and esophageal motility disorders $(5,6)$. The isolation of an NTM species from a respiratory sample is not sufficient evidence of the presence of NTM lung disease, the diagnosis of which is based on clinical, radiographic, and bacteriological criteria (1). In this study, we discussed five cases of NTM that were diagnosed and followed up at our clinic between 2008 and 2010.

\section{CASE PRESENTATIONS}

Case 1

A 65-year-old female patient with rheumatoid arthritis was admitted to our clinic because of complaints of cough, thoracic pain, and hemoptysis. She was taking leflunomide and methylprednisolone for six months. She had no history of smoking. Computerized tomography (CT) of the thorax showed mediastinal lymphadenopathy that had no pathological size and bilateral ground-glass appearance, consolidation areas, and multiple reticulonodular infiltrations some of which showed cavitation (Figure 1). Because sputum smears for acid-fast bacilli (AFB) were found positive two times, anti-TB treatment with isoniazid $(H)$, rifampicin $(R)$, ethambutol $(E)$, and pyrazinamide $(Z)$ was initiated. Mycobacterial resistance to HRE and streptomycin (S) was found. Two sputum smears at the beginning of TB treatment yielded positive cultures for NTM. M. abscessus was identified by polymerase chain reaction (PCR). It was sensitive to amikacin (AK), cefoxitin (CFX), tobramycin, and clarithromycin (CL). M. $a b$ scessus treatment with IV AK 750 mg/day, oral CL 1 g/day, IV CFX 2 g/day, and oral ciprofloxacin (CIP) $1 \mathrm{~g} /$ day was started. Seven months after the beginning of treatment, she died. 


\section{Case 2}

A 74-year-old man was hospitalized because of COPD exacerbation. Thorax CT showed reticular and nodular infiltrations, consolidations, bronchiectasis, ground-glass appearance at the left lower and upper lobe segments, consolidation, ground-glass appearance, reticular infiltrations at right lower lobe segments, cavitary infiltration $(1.5 \mathrm{~cm}$ in diameter), peribronchovascular reticulonodular infiltrations at right upper lobe segments, atelectasia and bronchiectasis at the middle lobe, and thrombus at the left main pulmonary artery and descending pulmonary artery (Figure 2). Three sputum smears were positive for AFB. Anti-TB treatment with HRSE was initiated. Sputum smear for AFB was negative three times at the second month of TB treatment, and the TB cultures were also negative. Anti-TB treatment finished six months later. Only one sputum smear at the second month of anti-TB treatment yielded positive culture for NTM. M. chelonae was identified by PCR. It was resistant to $H, R, E$, $\mathrm{S}$ and susceptible to ciproxin (CIP), capreomycin (CAP), kanamycin (KAN), and amikacin (AK). Treatment for M. chelonae was not initi-

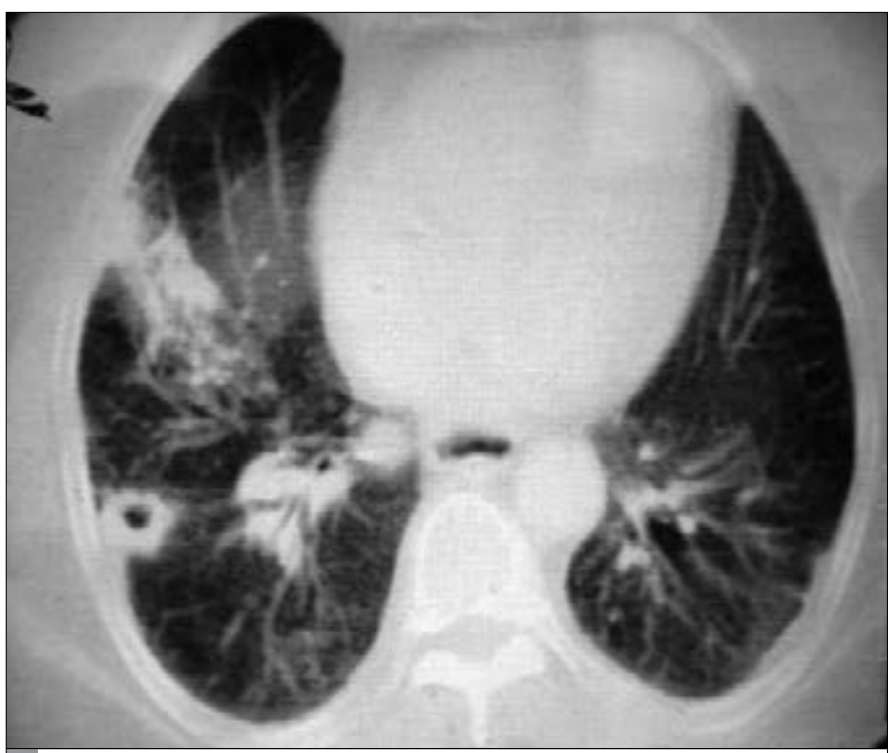

Figure 1. Bilateral ground glass appearence, consolidation areas, reticulonodular and cavitary infiltrations

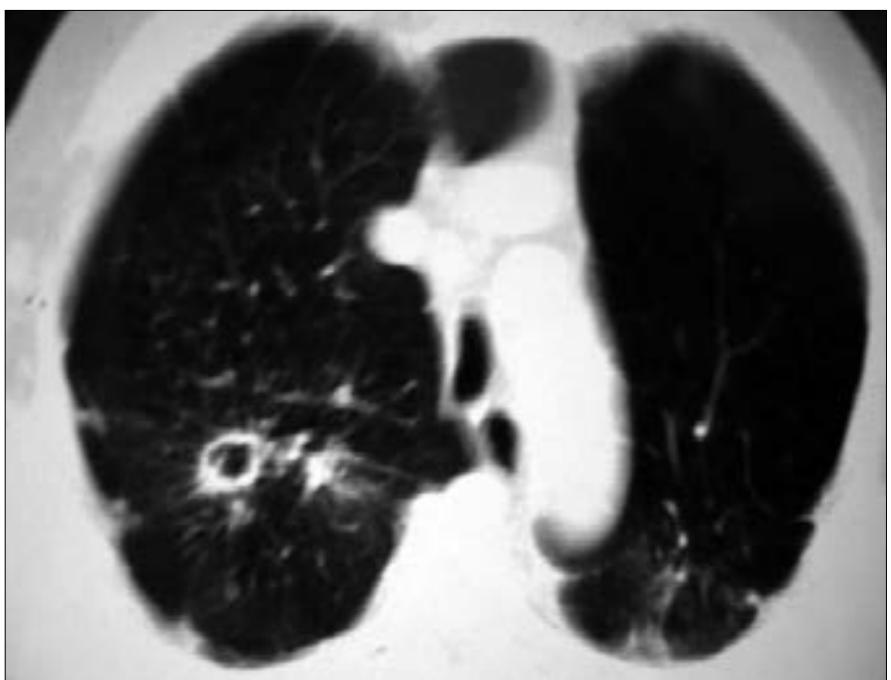

Figure 2. Reticular, nodular and cavitary infiltrations ated because only one sputum smear was positive for $M$. chelonae, and all other sputum smears were negative for AFB and other cultures. During the one-year follow-up without treatment for NTM, progression of disease was not seen.

\section{Case 3}

A 73-year-old man with coronary artery disease and diabetes mellitus (DM) was hospitalized with a complaint of fatigue. He was smoker. Thorax CT showed cavitary infiltrates in the right lung (Figure $3)$. Bronchial lavage smear was negative for AFB. Nonspesific antibiotic treatment was given for two weeks. However, there was no clinicoradiological regression. Anti-TB treatment with HRZE was initiated. The bronchial lavage smear yielded a positive culture for NTM. M. gordoneae was identified with PCR. It was resistant to $\mathrm{H}, \mathrm{R}, \mathrm{E}, \mathrm{S}$, cycloserine (CYC), ethionamide (ETN), and para-aminosalicylic asid (PAS) and was sensitive to ofloxacin (OFL), CIP, KAN, AK, and CAP. Treatment with oral linezolid $1200 \mathrm{mg} /$ day, CL 1g/day, and OFL $800 \mathrm{mg} /$ day was initiated. However, because the patient was not compliant, the treatment ended after 10 days. During the one-year follow-up without treatment, clinical and radiological progression did not occur.

\section{Case 4}

A 53-year-old man with complaints of cough and hemoptysis was admitted to our clinic. Chest radiography showed cavitary infiltration in the right upper lung zone and heterogeneous infiltrations in the left middle and lower lung zones. Thorax CT showed cavitary infiltration in the right lung and nodular infiltration in left lung (Figure 4). Three sputum smears were positive for AFB. Anti-TB treatment with HRZE was started. Two sputum smears at the beginning of anti-TB treatment yielded positive cultures for NTM. M. szulgai was identified. It was resistant to CYC, ETN, and PAS and was sensitive to $H, R$, E, S, OFL, CIP, ETN, CAP, KAN, and AK. Treatment for M. szulgai was continued with HRZE. One year after treatment, clinicoradiological regression occurred, and all sputum smears, AFB, and cultures for TB became negative.

\section{Case 5}

A 20-year-old man with DM was admitted to our clinic because of complaints of cough, sputum, and weight loss. Chest radiography showed pleural effusion appearing as a homogeneous opacity in

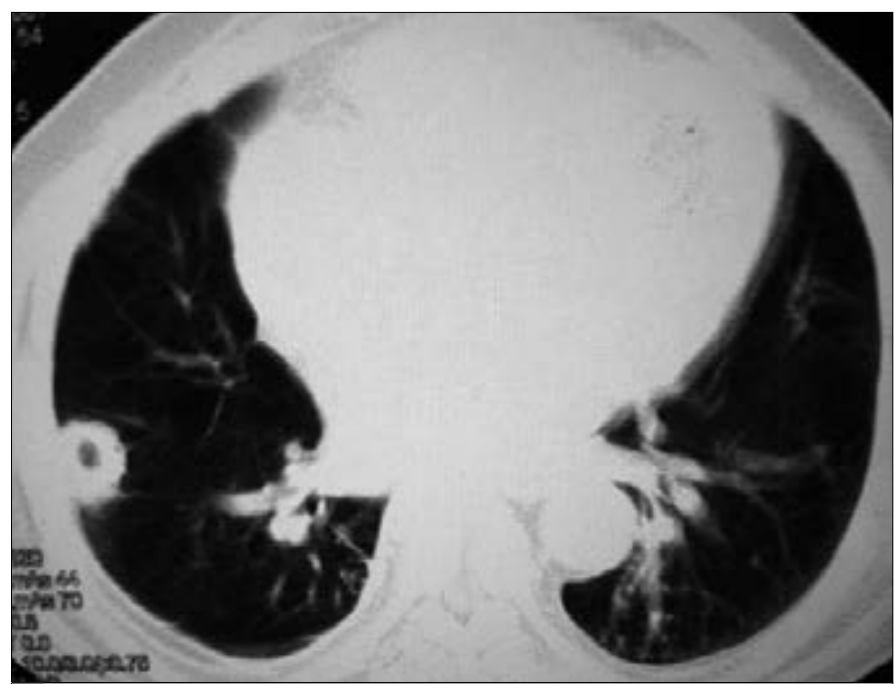

Figure 3. Thorax $\mathrm{CT}$ : Cavitary infiltrates in right lung 
the lower zone of the right lung. After thoracentesis, pneumothorax occurred. Chest tube thoracostomy was performed to drain air around the lungs. Then, sputum smears were found positive for AFB two times. Anti-TB treatment with HRZE was started with the diagnosis of pulmonary TB. Empyema occurred during drainage of the pleural space by the chest tube. Four months after treatment, pleural decortication was performed. Thorax $\mathrm{CT}$ showed consolidation and nodular infiltration in the right lung (Figure 5). Sputum smears were positive for AFB four months after treatment. Five sputum smears yielded positive cultures for NTM. M. abscessus was identified. It was resistant to HRES, CIP, doxycycline, imipenem, tobramycin, and trimethoprim and was sensitive to AK, CL, tigecycline, CFX. Treatment for M. abscessus was initiated with IV AK 1g/day, oral CL 1g/day, and IV CFX 2 g/day. However, because the patient did not want to continue treatment, it was stopped after two months. During the one-year follow up without treatment, clinical and radiological progression did not occur.

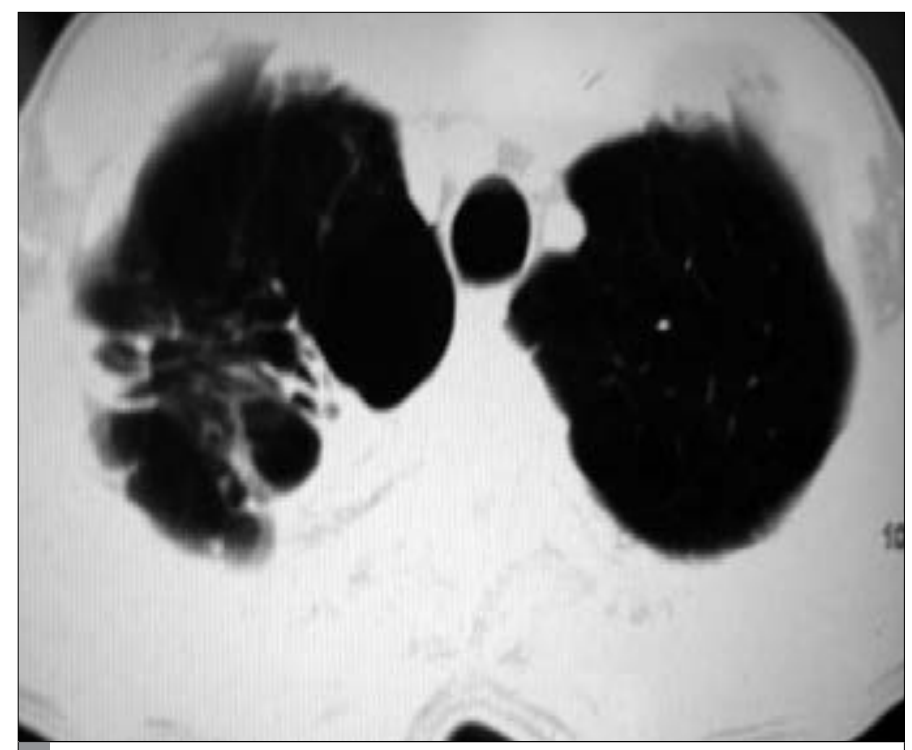

Figure 4. Cavitary infiltration and bronchiectasies in right lung

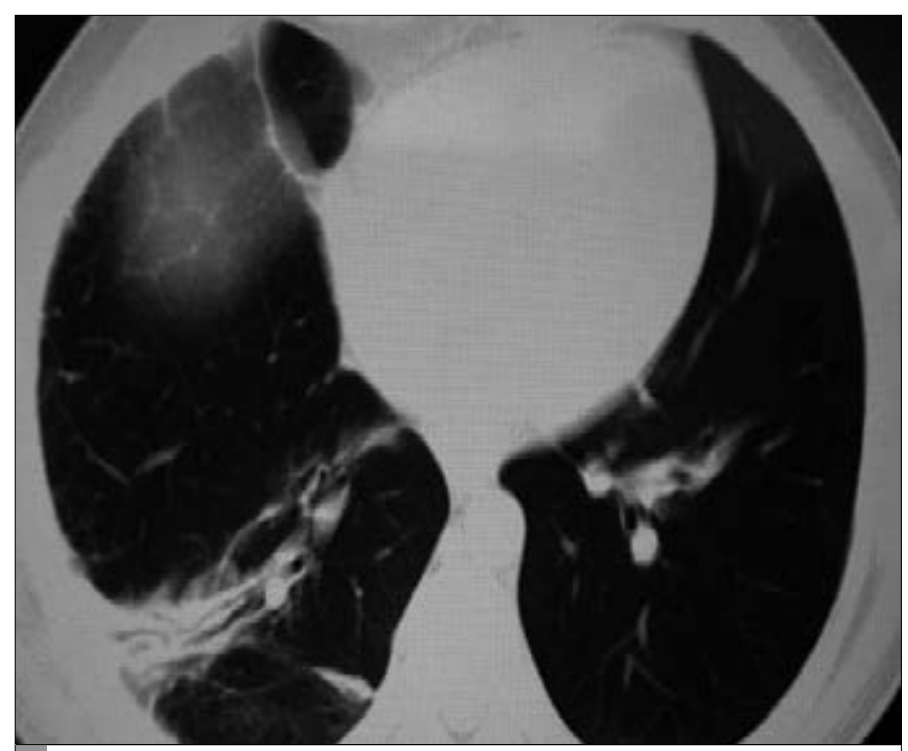

Figure 5. Consolidation and nodular infiltration in right lung

\section{DISCUSSION}

Although M. tuberculosis is by far the most important mycobacterial species from the public health perspective, other species are being encountered with an increasing frequency and new species are being identified (7). Unlike M. tuberculosis, which is an obligate human pathogen with no environmental reservoir, NTM are commonly isolated from environmental sources such as water and soil, and until the second half of the previous century, occasional isolates of NTM were thus largely considered to be contaminants or colonizers (1-4). Some NTM, however, are pathogens that may cause severe disease or even result in death $(1,2,8)$. The isolation of an NTM species from a respiratory sample is not sufficient evidence for the presence of NTM lung disease, the diagnosis of which is based on clinical, radiographic, and bacteriological criteria (1).

Person-to-person transmission of infection is rare, and isolation of infected individuals is thus not required. It is generally accepted that most human infection is due to environmental NTM and that airborne NTM infection may play an important role in respiratory diseases $(1,8)$.

Human diseases due to NTM is classified into four distinct clinical syndromes: pulmonary disease, lymphadenitis, cutaneous disease, and disseminated disease; among these, chronic pulmonary disease is the most common localized clinical condition $(1,8)$. Lung disease due to NTM commonly occurs in structural lung disease, such as COPD, bronchiectasis, cystic fibrosis, pneumoconiosis, prior TB, pulmonary alveolar proteinosis, and esophageal motility disorders (5).

The isolation of NTM species from a respiratory sample is not sufficient evidence of the presence of NTM lung disease, the diagnosis of which is based on clinical, radiographic, and bacteriological criteria (1). The minimum evaluation of a patient suspected of having NTM lung disease should include the following: 1) chest radiograph or, in the absence of cavitation: 2) three or more sputum specimens for AFB analysis, and 3) exclusion of other disorders such as TB (1). Clinical, radiographic, and microbiological criteria are equally important, and all must be met to make a diagnosis of NTM lung disease (1).

The radiological manifestations of NTM pulmonary disease vary and are often subtle and may be indistinguishable from those of TB. The radiographic criteria required are the presence of infiltrates, cavitation, or multiple nodules on plain chest radiography and/or multiple small nodules less than $10 \mathrm{~mm}$ in diameter or multifocal bronchiectasis on high-resolution computed tomography (HRCT) of the lungs (9). Koh et al. (10) suggested that approximately one-third of patients with bilateral bronchiectasis and bronchiolitis have NTM disease extensive abnormalities, cavitation, or consolidation on HRCT and that females were particularly associated with NTM. Radiographic features of NTM lung disease depend on whether the lung disease is primarily fibrocavitary (similar to TB) or characterized by nodules and bronchiectasis (nodular/bronchiectatic disease) (5).

In the absence of diagnostic specificity of the clinical manifestations or chest radiographic findings, the diagnosis of NTM pulmonary disease requires microbiological confirmation. Positive sputum cultures for NTM should, however, be cautiously interpreted. The discovery of NTM in a single sputum sample is not proof of NTM disease, especially when the AFB smear is negative and NTM are cultured in small numbers. The distinction between colonization or 
contamination and true infection is often difficult and somewhat arbitrary $(5,9)$.

According to theATS/IDSA statement (2007), the following criteria applied to make a diagnosis of NTM lung disease: 1) pulmonary symptoms, chest radiograph, 2) positive culture results from at least two separate expectorated sputum samples or positive culture results from at least one bronchial lavage, or 3) transbronchial or other lung biopsy with mycobacterial histopathological features and positive culture for NTM or biopsy showing mycobacterial histopathological features and one or more sputum or bronchial washings that are positive for NTM (5). Patients who are suspected of having NTM lung disease but who do not meet the diagnostic criteria should be followed up until the diagnosis is firmly established or excluded. Making the diagnosis of NTM lung disease does not, per se, necessitate the administration of therapy, which is a decision based on the potential risks and benefits of therapy for individual patients. In developing countries where the incidence of pulmonary TB is much higher than that of NTM pulmonary disease, the initiation of presumptive anti-TB treatment, especially in smear-positive patients prior to the identification of isolates, is a common practice (9).

All the five cases in this study had pulmonary TB like clinicoradiological appearance. Four cases had sputum smears positive for AFB, and one case had a positive bronchial culture for NTM. Radiological findings of our five cases were consolidations, cavitary infilrations, nodular infiltrations, and bronchiectasia. These findings were similar with the study of Babalık (11). Anti-TB treatment was started for all cases.

All isolates of NTM except M. szulgai were resistant to HRSE. One patient with $M$. abscessus, despite aggressive treatment, died, whereas the other was alive and had no clinical and radiological progression without treatment. Without treatment, progression of disease was not seen in the case of $M$. chelonae and that of $M$. gordoneae. Clinicoradiological regression occurred in the case of M. szulgai that was treated with HRZE.

Limitations of this study are that a small number of subjects were used and that there was a treatment noncompliance in two cases.

\section{CONCLUSION}

As a conclusion, NTM have pulmonary TB like clinicoradiological appearance and sputum smears positive for AFB. Anti-TB treatment should be started for all cases. When there is TB drug resistance, the possibility of NTM should be kept in mind. For correct diagnosis and the successful treatment of NTM pulmonary disease, knowledge of clinical, radiological, and microbiological findings is important.

Peer-review: Externally peer-reviewed.

Author contributions: Concept - M.G., A.Ş.; Design - A.Ş., M.G.; Supervision - E.U., N.Ç.; Resource - A.Ş., R.O.; Materials - M.G., A.Ş.; Data Collection and/or Processing - M.G., A.Ş.; Analysis and/or Interpretation - M.G., A.Ş.; Literature Search - A.Ş., R.O.; Writing - A.Ş., M.G.; Critical Reviews - E.U., N.Ç.; Other - M.G., A.Ş.

Conflict of Interest: No conflict of interest was declared by the authors.

Financial Disclosure: The authors declared that this study has received no financial support.

\section{REFERENCES}

1. Diagnosis and treatment of disease caused by nontuberculous mycobacteria. This official statement of the American Thoracic Society was approved by the Board of Directors, March 1997. Medical Section of the American Lung Association. Am J Respir Crit Care Med 1997; 156: S1-S25.

2. Wolinsky E. Nontuberculous mycobacteria and associated diseases. Am Rev Respir Dis 1979; 119: 107-59.

3. Falkinham JO 3rd. Nontuberculous mycobacteria in the environment. Clin Chest Med 2002; 23: 529-51. [CrossRef]

4. Glassroth J. Pulmonary Disease due to nontuberculous mycobacteria. Chest 2008; 133: 243-51. [CrossRef]

5. Griffith DE, Aksamit T, Brown-Elliott BA, Catanzaro A, Daley C, Gordin F, et al. An official ATS/IDSA statement: diagnosis, treatment, and prevention of nontuberculous mycobacterial diseases. Am J Respir Crit Care Med 2007; 175: 367-416. [CrossRef]

6. Field SK, Cowie RL. Lung disease due to more common nontuberculous mycobacteria. Chest 2006; 129: 1653-72. [CrossRef]

7. Tortoli E. Impact of genotypic studies on mycobacterial taxonomy: the new mycobacteria of the 1990s. Clin Microbiol Rev 2003; 16: 319-54. [CrossRef]

8. Management of opportunist mycobacterial infections: Joint Tuberculosis Committee Guidelines 1999. Subcommittee of the Joint Tuberculosis Committee of the British Thoracic Society. Thorax 2000; 55: 210-8. [CrossRef]

9. Koh WJ, Kwon OJ, Lee KS. Nontuberculous mycobacterial pulmonary diseases in immunocompetent patients. Korean J Radiol 2002; 3: 145-57. [CrossRef]

10. Koh WJ, Leek S, Kwon OJ, Jeong YJ, Kwak SH, Kim TS. Bilateral bronchiectasis at thin-section $\mathrm{CT}$ : diagnostic implications in nontuberculous mycobacterial pulmonary infection. Radiology 2005; 235: 282-8. [CrossRef]

11. Babalık A, Kuyucu T, Ordu EN, Ernam D, Partal M, Köksalan K. Non-tuberculous mycobacteria infection: 75 cases. Tuberk Toraks 2012; 60: 20-31. [CrossRef] 